\title{
Screening Utility, Local Perceptions, and Care-seeking for Reported Jaundeesh among Respondents Lacking Signs of Icterus in Rural Bangladesh
}

\author{
Mohammad Z. Hossain', Shegufta S. Sikder², K. Zaman', Parimalendu Saha', \\ Mohammad Yunus', Kenrad E. Nelson ${ }^{3}$, Alain B. Labrique ${ }^{2,3}$ \\ 'icddr,b, GPO Box 128, Dhaka 1000, Bangladesh; ${ }^{2}$ Department of International Health, ${ }^{3}$ Department of Epidemiology, Johns \\ Hopkins Bloomberg School of Public Health, 615 N. Wolfe St., Baltimore, MD 21205, USA
}

\begin{abstract}
In rural Bangladesh, acute viral hepatitis presents a significant burden on the public-health system. As part of the formative work for a large epidemiologic study of hepatitis $\mathrm{E}$ in rural Bangladesh, we sought to identify local terms that could be used for population-based screening of acute viral hepatitis. Exploration of the local term jaundeesh for screening utility identified a high burden of reported jaundeesh among individuals without symptoms of icterus. Recognizing that local perceptions of illness may differ from biomedical definitions of disease, we also sought to characterize the perceived aetiology, care-seeking patterns, diagnostic symptoms, and treatments for reported jaundeesh in the absence of icteric symptoms to inform future population-based studies on reported morbidities. We conducted a cross-sectional survey among 1,441 randomly-selected subjects to identify the prevalence of reported jaundeesh and to test the validity of this local term to detect signs of icterus. To characterize the perceived aetiology and care-seeking patterns for jaundeesh among the majority of respondents, we conducted in-depth interviews with 100 respondents who self-reported jaundeesh but lacked clinical signs of icterus. To describe diagnostic symptoms and treatments, in-depth interviews were also performed with 25 kabirajs or traditional faith healers commonly visited for jaundeesh. Of the 1,441 randomly-selected participants, one-fourth $(\mathrm{n}=361)$ reported jaundeesh, with only a third $(n=122)$ reporting yellow eyes or skin, representative of icterus; Jaundeesh had a positive predictive value of $34 \%$ for detection of yellow eyes or skin. Anicteric patients with reported jaundeesh perceived their illnesses to result from humoral imbalances, most commonly treated by amulets, ritual handwashing, and bathing with herbal medicines. Jaundeesh patients primarily sought folk and spiritual remedies from informal care providers, with only $19 \%$ visiting allopathic care providers. Although the local term jaundeesh appeared to have limited epidemiologic utility to screen for acute symptomatic viral hepatitis, this term described a syndrome perceived to occur frequently in this population. Future population-based studies conducting surveillance for acute hepatitis should use caution in the use and interpretation of self-reported jaundeesh. Further study of jaundeesh may provide insight into the appropriate public-health response to this syndrome.
\end{abstract}

Key words: Ethnography; Hepatitis; HEV; Jaundice; Medical anthropology; Morbidity; Traditional healers; Bangladesh

\section{INTRODUCTION}

Viral hepatitis is a significant public-health problem in Bangladesh (1). Although hepatitis A and $\mathrm{E}$ viruses (both water-borne, faecal-oral pathogens)

Correspondence and reprint requests:

Dr. Alain B. Labrique

Johns Hopkins Bloomberg School of Public Health

615 N. Wolfe St., E5543

Baltimore, MD 21205

USA

Email: alabriqu@jhsph.edu cause low mortality in the general population, these lead to significant annual morbidity and loss of productivity across much of South Asia $(1,2)$. Exposure to hepatitis A is nearly ubiquitous, resulting in widespread infection in early life and consequent lifelong immunity (1). Assessing clinical data, 1,823 patients in Dhaka, Bangladesh, suspected to suffer from hepatitis B, Khan et al. detected antibodies to hepatitis A and E virus among 39\% and $53 \%$ of subjects respectively. Hepatitis E, associated with elevated case-fatality ratios in pregnant 
women (up to 20\%) (3), is considered a major public-health problem across most of South Asia and has been shown to contribute up to $10 \%$ of pregnancy-related deaths in rural northwest Bangladesh $(4,5)$. Despite this burden, this emerging pathogen remains significantly under-studied and under-recognized as an aetiologic agent in this population $(3,6)$. The ubiquitous risk of exposure to faecal-oral pathogens has been well-documented across rural and urban populations of the country, explaining the elevated burden of viral hepatitis infections $(3,6)$.

Improved contextual understanding of specific diseases in endemic populations is essential to optimize surveillance, control, and targeted care. In settings where clinical consultations may be challenging, population-based surveys seek to elicit reported symptoms and morbidities during routine surveillance. For detection of acute viral hepatitis, population-based surveys often assess report of clinically-proven jaundice, also known as icterus (the yellowing of the eyes or skin as a result of hyperbilirubinaemia or liver cholestasis-a sign of liver distress or damage) as a symptom that triggers further clinical investigation for presence of hepatitis (7). While local terms may be useful in detecting symptoms warranting further investigation, studies in rural Bangladesh have suggested that local perceptions of illness may differ from biomedical definitions (8-11). For conditions ranging from infectious and chronic diseases to pregnancy-related illnesses, studies have described cultural frameworks of perceived illness aetiology, which influence conceptions of disease as well as subsequent care-seeking behaviours (8-12). Despite the documented elevated burden of viral hepatitis, little has been reported about the medical anthropology of this spectrum of infections, including local perceptions and practices, care-seeking behaviour, and treatments.

In Bangladesh, perceived aetiologies of illness have been linked to care-seeking behaviours $(8-10,13)$. Studies from Bangladesh reveal a plural health system that consists of both informal (defined as care providers lacking formal certification) and formal care providers (defined as healthcare providers who are recognized and regulated by legal authorities), such as doctors, nurses, midwives, or government-trained care providers $(8-10,13)$. For illnesses, including some that are typically believed to arise from non-medical causes, rural residents have reported visiting informal care providers, such as traditional healers, village doctors, shamans, traditional birth attendants, and homeopathic doctors (14). Traditional healers, including shamans, are generally visited for conditions that are perceived to be caused by evil spirits, and they provide blessings against these spirits (15). In Bangladesh, a common traditional healer is called kabiraj, a healthcare provider who practises a combination of ayurvedic medicine and faith healing (15). Allopathic care providers include village doctors and homeopathic care providers, some with training or apprenticeship experience, who provide allopathic treatments for various ailments in rural communities $(9,16)$. Although studies reveal the importance of informal care providers in care-giving for numerous illnesses, care-seeking for hepatitis and hepatitis-like illnesses has not been well-described in this rural population. A recent large outbreak of hepatitis $\mathrm{E}$ in an urban population of Bangladesh nearly went undetected by the public health sector (Gurley. Personal Communication, 2010) as most of the cases did not seek formal care for their condition. Although understanding care-seeking pathways is critical to developing effective public-health strategies for prevention and treatment (10), data on perceptions of jaundice in rural communities are lacking.

As part of the formative research for a large epidemiologic study of hepatitis E in rural Bangladesh, we sought to test the validity and utility of local terms tested and validated elsewhere in the country, to detect clinical signs of icterus in this population. The frequent report of the local term jaundeesh was initially a promising finding, given the strong pathognomonic association of scleral and dermal icterus with acute hepatitis (7). However, formative research suggested a high burden of reported jaundeesh among respondents without symptoms of icterus (6). Recognizing that local illness perceptions may differ from biomedical definitions of disease, we also sought to characterize the perceived aetiology, care-seeking patterns, diagnostic symptoms, and treatments for reported jaundeesh in the absence of icteric symptoms to inform future population-based studies on reported morbidities.

\section{MATERIALS AND METHODS}

The study was conducted in Matlab (a subdistrict approximately $55 \mathrm{~km}$ southwest of Dhaka) where the International Centre for Diarrhoeal Disease Research, Bangladesh (icddr,b) has been implementing a health and demographic surveillance system in a population of about 220,000 for the past four decades (17). Since 1966, community health research workers (CHRWs) have collected vital statistics, such as on marriage, birth, death, and migration among this population. 
This three-phase study was conducted as part of the formative research preceding a large longitudinal study which sought to identify age-specific incidence of hepatitis $\mathrm{E}$ infections under endemic, non-outbreak conditions in rural Bangladesh (3). The formative research, completed in 2003, was conducted in three phases to achieve the three research objectives. From the 2003 Census of the Matlab Health and Demographic Surveillance System (HDSS), a random list of 1,450 individuals was generated for inclusion. Children aged less than one year were not sampled due to the reluctance of parents to allow blood-draws from healthy young infants.

From the selected 1,450 individuals, 1,441 consented for hepatitis surveillance and were included in this study. Thirteen CHRWs regularly visited households to ask participants if, in the last 30 days, they or anyone in their family experienced any of the following six hepatitis-like symptoms: (i) nausea or vomiting, (ii) fever and severe weakness, (iii) loss of appetite or foul smell in food, (iv) yellow eyes or skin, (v) ash-coloured stool or tea-coloured urine, or (vi) jaundeesh/holde palong. Pre-testing of these questions revealed that the local terms jaundeesh or holde palong were sometimes used for describing conditions consistent with biomedical jaundice, these terms were used in the screening algorithm at the outset of the study. If respondents reported experiencing any symptoms, the duration of each reported symptom was recorded.

If participants reported experiencing jaundeesh/ holde palong lasting between eight and 14 days, the CHRWs sent their information to a medical team (consisting of an MBBS doctor and a laboratory technician) to schedule an examination. A blood sample was collected if physicians found the participant to exhibit visible signs consistent with clinical icterus. For practical purposes, only those respondents who had visible icterus, defined as yellow eyes or skin confirmed by a medical officer, were approached for blood samples. The medical teams used the symptoms of yellow eyes or skin for icterus due to greater practicality of confirming these symptoms during home-visits compared to symptoms, such as ash-coloured stool or teacoloured urine. In addition, the symptoms of dark-coloured urine or light clay-coloured stools have been considered to be less reliable for detection of hepatitis compared to the symptoms of yellow eyes or skin (18).

The positive predictive value and negative predictive value of the terms jaundeesh/holde palong to identify patients with yellow eyes or skin were calculated. Data from this process led to further refinement of the screening algorithm and the eventual exclusion of jaundeesh/holde palong. Findings from the larger study, published elsewhere, showed that most of the tested participants did not exhibit acutephase antibodies to hepatitis A, B, C, or E (3).

Recognizing the large burden of patients who reported jaundeesh/holde palong in this population in the absence of visible signs of icterus, we sought to further characterize these local terms by describing their common perceived aetiology, diagnostic symptoms, and treatments to inform future population-based studies. The second phase of formative research consisted of in-depth interviews with100 participants, randomly selected from respondents who self-reported jaundeesh and who lacked symptoms of yellow eyes or skin. Two field research assistants, both with five years of experience implementing field interviews in Matlab and with training on qualitative methods, conducted in-depth interviews, using a structured questionnaire. The field research assistants asked respondents to describe their understanding of how their jaundeesh was acquired, the symptoms that they associated with this condition, and their typical treatmentseeking behaviour.

Respondents were asked about the healthcare providers from whom they typically sought treatment for themselves or their family members. Since kabirajs were the most commonly-visited treatment providers, the interview team asked respondents about the name and location of the kabirajs that they visited. Using this list for the third stage of formative research, the team conducted in-depth interviews with the 25 most commonly-visited traditional healers or kabirajs in the Matlab study area to understand how these care providers diagnosed jaundeesh and the treatments they commonly prescribed. The interview team asked the kabirajs about their age, years of experience, weekly number of patients seen, and diagnostic symptoms and treatments provided for jaundeesh/holde palong.

All quantitative data were analyzed in Excel 2010 to calculate sensitivity, specificity, positive predictive value, and negative predictive value of the term jaundeesh to detect symptoms of yellow eyes or skin. For the qualitative data from in-depth interviews, a medical officer and a senior field research officer assisted the field research assistants with transcription. Using Microsoft Word, the in-depth interviews were reviewed and coded line-by-line to 
identify common themes described as constituting jaundeesh. These data were used in producing a conceptual framework of aetiology of perceived jaundice in the study population. Quantitative data on care providers sought and their typical treatments were analyzed in Excel 2010 to produce frequency distributions of care providers most frequently approached, diagnostic symptoms, and common treatments. Study procedures were approved by the Committee on Human Research at the Johns Hopkins Bloomberg School of Public Health and by Ethical Review Committee of icddr,b.

\section{RESULTS}

\section{Prevalence and screening utility of reported jaundeesh}

Of the 1,441 people randomly selected for hepatitis surveillance, 361 (25\%) reported having jaundeesh while 1,080 people (75\%) reported that they did not have jaundeesh. Among the 361 people reporting jaundeesh, $84 \%$ reported loss of appetite or foul smell in food while $68 \%$ reported fever or severe weakness, and 51\% reported nausea or vomiting (Table 1). Only one-third of the respondents with jaundeesh (34\%) reported having yellow eyes or skin while $27 \%$ reported ash-coloured stool or teacoloured urine.

\begin{tabular}{|c|c|}
\hline \multicolumn{2}{|c|}{$\begin{array}{l}\text { Table 1. Hepatitis-like symptoms reported among } \\
361 \text { respondents who self-reported jaun- } \\
\text { deesh in Matlab, Bangladesh, in } 2003\end{array}$} \\
\hline Symptom & $\begin{array}{l}\text { Number (percentage) } \\
\text { reporting symptoms } \\
\text { among respondents who } \\
\text { self-reported jaundeesh/ } \\
\text { holde palong }(\mathrm{n}=361)^{\star}\end{array}$ \\
\hline $\begin{array}{l}\text { Loss of appetite and } \\
\text { foul smell in food }\end{array}$ & $304(84)$ \\
\hline $\begin{array}{l}\text { Fever and severe } \\
\text { weakness }\end{array}$ & $245(68)$ \\
\hline Nausea or vomiting & $185(51)$ \\
\hline Yellow eyes or skin & $122(34)$ \\
\hline $\begin{array}{l}\text { Ash-coloured stool or } \\
\text { tea-coloured urine }\end{array}$ & 99 (27) \\
\hline
\end{tabular}

Table 2 shows the positive predictive value of the term jaundeesh/holde palong to detect yellow eyes or skin. The positive predictive value of the terms jaundeesh/holde palong for detection of yellow eyes or skin was only $34 \%$, with only 122 out of 361 respondents with jaundeesh reporting yellow eyes or skin (Table 2). The negative predictive value of the terms was relatively high since among those not reporting jaundeesh/holde palong, 99\% $(n=1,071)$ did not report yellow eyes or skin. Although 93\% of respondents with yellow eyes or skin reported jaundeesh (Table 2), the term jaundeesh had limited screening utility to effectively capture icterus, primarily due to the dilution of this pool with a large number of individuals without visible symptoms of yellow eyes or skin.

\begin{tabular}{|c|c|c|c|c|}
\hline & & Yel & $\checkmark$ eyes $\mathrm{O}$ & skin \\
\hline & & Yes & No & Total \\
\hline Jaundeesh/ & Yes & 122 & 239 & 361 \\
\hline holde & No & 9 & 1,071 & 1,080 \\
\hline palong & Total & 131 & 1,310 & 1,441 \\
\hline & Sens & ity: 93 & & \\
\hline & Spec & ity: 82 & & \\
\hline & ive pre & tive $\mathrm{v}$ & e: $34 \%$ & \\
\hline $\mathrm{N}$ & tive pr & tive $\mathrm{v}$ & de: $99 \%$ & \\
\hline
\end{tabular}

Perceptions of jaundeesh among those lacking visible symptoms of icterus

Field research assistants conducted in-depth interviews with 100 randomly-selected respondents to understand local perceptions of jaundeesh among anicteric respondents. Figure 1 presents a framework of how jaundeesh is commonly believed to be manifested, based on common responses. Respondents mentioned eating at incorrect times, insufficient water intake, and hard work in the sun contributing to humoral imbalances, such as bile (local term: pitta) becoming hot or the body 'drying up' and constipation. Symptoms described as indicative of holde palong included a burning sensation in hands and feet, fever, foul smell in food or water, anorexia, weakness, yellow eyes or urine, and nausea. Ranu, a 33-year old woman, explained the process of jaundice acquisition:

Village people say when the body dries up, when the hands and feet burn, stool hardens, and urination decreases, jaundeesh happens. Then, many people bathe in their ponds two to three times a day for at least an hour. When the body gets cold, your nose starts to run, then the jaundeesh gets better.

Mahfuza, a 40-year old woman, explained a similar process:

Because jaundeesh happens from constipation, it means the body, hands, and feet burn, stool 


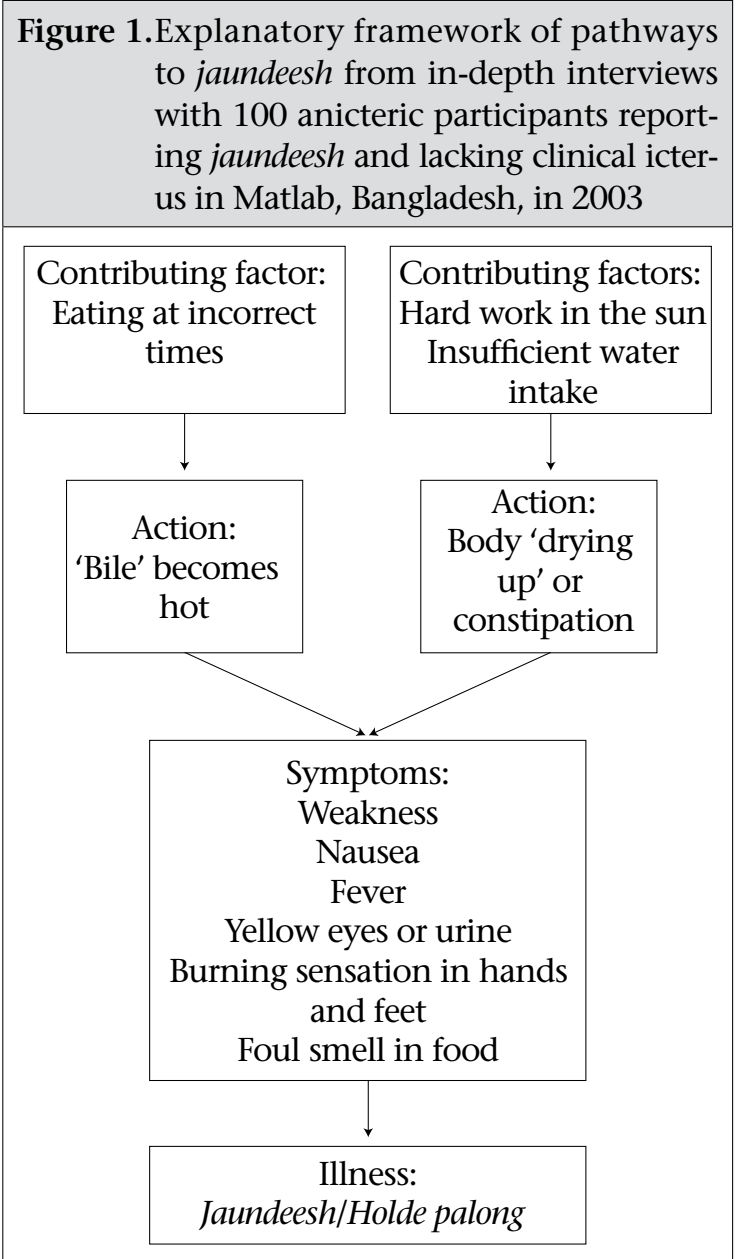

becomes hard and ashy. This is why, in the village, those who have jaundeesh bathe every hour in the pond. When it is a child, they bathe him [or her] three to four times. They [people with jaundeesh] walk around without shoes, so that they get cold. When you get cold, your jaundice is cured.

Most people believed that they had jaundeesh due to symptoms, such as loss of appetite, fever, or nausea. Rima, a 30-year old mother who reported Jaundeesh in her 3-year old son, explained:

Last monsoon, my son had jaundeesh. When I fed him, he would act nauseated and sometimes would vomit. He had fever, and his stool was hard. He didn't want to eat anything. When I saw these symptoms, I realized my son had jaundeesh.

Aklima, a 29-year old female, describes similar symptoms:

When I was sick, I had fever and nausea. I could not eat anything; everything had a smell of fish. I had a burning sensation in my hands and feet. Then, I realized I had jaundeesh.

Care-seeking for jaundeesh among those lacking visible symptoms of icterus

Figure 2 shows that $59 \%$ of the respondents sought care from traditional treatment providers called kabiraj while $19 \%$ went to village doctors, and $11 \%$ visited shamans, or spiritual healers. Seven percent sought no treatment while the remaining 4\% sought treatment from both a kabiraj and an MBBS (board-certified) doctor.

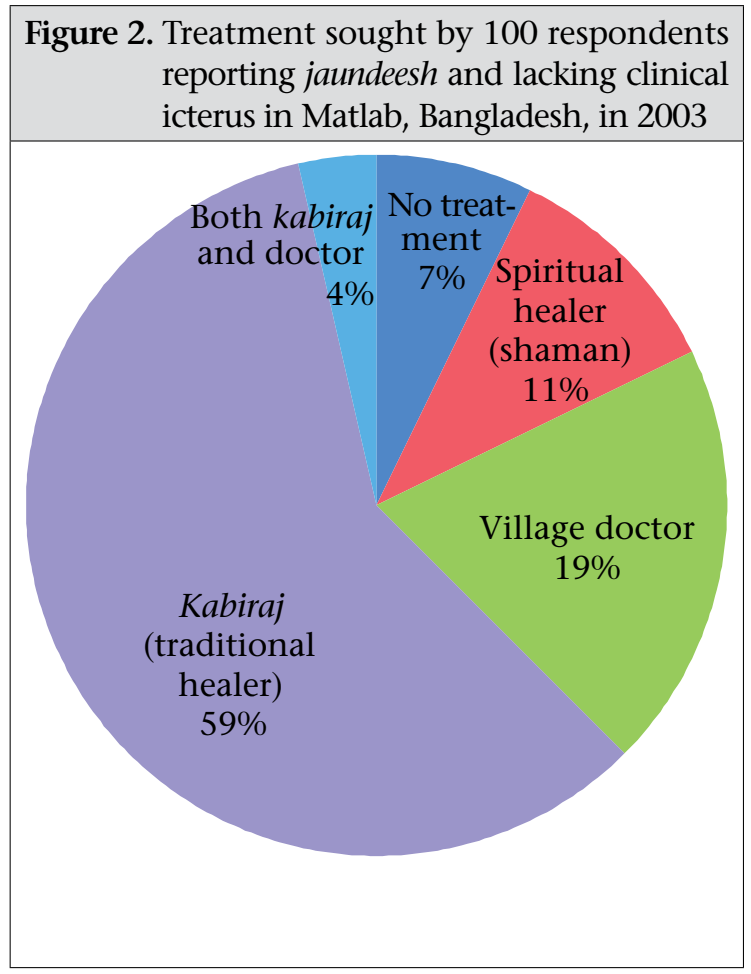

From the in-depth interviews, respondents described visiting kabirajs since they believed the treatments would cure their jaundeesh.

Shanti, a 26-year old woman with jaundeesh said:

I believe that treatment from kabiraj is the best for jaundeesh. When I had jaundeesh, the kabiraj told me to eat blessed bananas for four days. After clean-shaving the top of my head, the kabiraj applied herbal paste on my scalp, which I kept for seven days. I also took herbal tablets three times a day for one month, and my jaundeesh was cured.

Respondents who sought care from both kabirajs and MBBS doctors said they did so due to the lack of specific treatments provided by allopathic doctors. Many of the respondents were pregnant and were not given specific treatments from their doctors. 
Jharna, a 23-year old woman, explained:

When I realized I had jaundeesh, my father-inlaw and husband took me to the hospital. As I was at the seventh month of my pregnancy, they did not give me any medicine and told me to go home and rest. When I came home, everyone told me to see the kabiraj for treatment. The kabiraj clean-shaved the top of my head and applied herbal paste. For three days, I went to his house so he could wash my hands in 'root-water' and place an amulet on my neck. After one and a half months, my symptoms were gone.

Parveen, 27-year old woman, described:

When I had jaundeesh, I went to the hospital. They gave me no treatment since I was pregnant and told me to drink lots of fluids. I went to the kabiraj who rubbed my hands with branches and leaves and then washed them. With his treatment, my jaundeesh disappeared in three months. Some kabirajs do handwashing, others give blessed bananas, and some others give amulets to wear. This is the way jaundeesh is cured.

Others went to kabirajs because they received greater satisfaction from their treatment compared to allopathic treatment. Mother of Abu Bakr, a 3-year old boy with reported jaundeesh said:

In the hospital, the doctors did blood tests and said my son had jaundeesh. They gave him vitamin drops and told us to come back in four days. I did not go back since my child was not improving with hospital treatment. Instead, I took my child to a kabiraj. The kabiraj fed my son one blessed banana every morning for three days. He also fed him the juice from tree leaves. After one and a half months, my son was completely cured. To cure my child, I had to visit the kabiraj, and he got better treatment compared to the hospital.

\section{Diagnostic symptoms and common treatments for jaundeesh}

Since kabirajs were the most commonly-visited treatment providers, the interview team conducted in-depth interviews with 25 most commonlyvisited kabirajs in the Matlab study area to understand symptoms used in diagnosing jaundeesh and the prescribed treatments. On average, the interviewed kabirajs were 54 years old, had been practising for 10 years, and provided treatment for jaundeesh to three patients per week. Of the 25 kabirajs interviewed, 48\% recorded patients' reports of foul smell in food and water as the main symptom that they used in diagnosing jaundeesh/ holde palong. Loss of appetite was the main diagnostic symptom used by one-fifth of the kabirajs while another 20\% cited dark-coloured urine. Only 12\% mentioned yellow eyes or skin as the main diagnostic symptom of jaundeesh.

When asked about the types of treatments they prescribed for jaundeesh, more than half described ritual handwashing procedures known as hat dhoa. As described by a 44-year old kabiraj who had been practising for seven years, "Hat dhoa means actively washing out jaundeesh, using herbal medicines, such as tree bark." During ritual handwashing, the kabirajs explained that yellow colouring was washed from the patients' hands to remove the offending jaundeesh with water. A 60-year old kabiraj who had been practising for 14 years explained, "Patients said that they prefer hat dhoa since they directly see the yellow being washed away and the jaundeesh coming out in front of them."

Table 3 shows the prescribed rituals and treatments for jaundeesh. Approximately one-fourth of the kabirajs recommended that patients wear sacred amulets around their neck, such as garlands or blessed items, to cure holde palong. One-fifth conducted ritual bathing of the body to remove jaundeesh. For children with jaundeesh, a 63-year old kabiraj who had been practising for 21 years explained:

Our wise elders say you must get up at dawn and dip the child in water every hour or so, or bathe the child in the pond 4-5 times a day so that he catches a cold. When he gets a cold (sneezing, runny nose), then you understand that the jaundeesh is running away.

Kabirajs commonly suggested that patients take herbal tablets or drink herbal mixtures. They described a variety of blessing rituals which they performed for their patients. Often, they performed a daily ritual at dawn at their home or their patient's home. For example, a 52-year old kabiraj who had been practising for 11 years explained his treatment process for children:

At dawn, I visit the sick child. After shaving the top of the head, I apply herbal paste at the top of the head every morning at dawn. I do this for three weeks to remove the jaundeesh. 
Table 3. Typical treatments prescribed by kabirajs $(\mathrm{n}=25)$ for jaundeesh/holde palong in Matlab, Bangladesh, in 2003

Description of ritual

Percentage of kabirajs recommending this treatment ${ }^{*}$

Wash hands with herbal medicines or mixtures $56 \%$

Wash out 'yellow' from patient's hands ('hat dhoa')

Wash patient's hands with tree bark

Wash hands with leaves from a vine ('lottapatha')

Wash hands with water that has been standing overnight

Wear amulet

Twist garland ('mala') on head/neck

Tie a banana with string, burn the banana, and tie the string to the patient's neck

Perform bathing ritual

Bathe with cold water until one gets a head cold to chase away jaundice

Dunk patient in water repeatedly until nose begins to run

Drink or apply herbal mixture

Drink water in which a live mussel has been soaked overnight

Shave head with a seashell and apply herbal paste

Drink unpasteurized milk

Consume symbolic item to remove jaundice

$16 \%$

Drink blessed water (panipora)

Eat blessed bananas (kolapora)

Eat blessed green coconut (dabpora)

Eat blessed sour yoghurt (dodhipora)

Place blessed paper-cone in ear and burn the cone

*The total is greater than $100 \%$ since more than one treatment could be listed

\section{DISCUSSION}

In the setting as ours in rural Bangladesh, onefourth of surveyed respondents perceived having a condition they called jaundeesh. Two-thirds of these respondents lacked the visible sign of yellow eyes or skin, suggestive of clinical icterus. Despite reasonably-high sensitivity and specificity, the term jaundeesh, in this rural Bangladesh context, provided limited positive predictive value due to the high burden of self-reported jaundeesh among those lacking yellow eyes or skin. Respondents perceived jaundeesh to arise from humoral imbalances and largely sought for non-biomedical treatments from kabiraj, particularly methods which claimed to 'wash out' the jaundeesh from their skin. As the main treatment providers in this population, only $12 \%$ of kabirajs used the symptom of yellow eyes or skin to diagnose jaundeesh.

Nearly two-thirds of the respondents with jaundeesh reported visiting kabirajs for care. Previous studies in rural Bangladesh indicate that patients may seek local treatment providers, such as kabirajs due to their accessibility, lower cost, and flexibility in payment schemes (10). One study conducted on informal treatment providers in Matlab, Bangladesh, in 1978 suggested that patients reporting jaundice almost exclusively visited kabirajs and other traditional healers for care, reflecting that these care-seeking pathways may, perhaps, be deeply ingrained in rural populations (11). Most kabirajs are deeply embedded in rural communities and are called to visit homes for treatment, reflecting a high degree of community trust. Kabirajs generally prescribe the use of herbal remedies which have been used since at least $175 \mathrm{BC}$ for treatment of jaundice in the Indian Subcontinent (19). Practices of faith healing rooted in ancient Animist religions and Pagan folk culture, and in rural areas, have been integrated into modern religious paradigms (15). Items believed to ward off evil spirits, such as amulets, local tree leaves, cow bones, iron knives, bamboo broomsticks, are commonly prescribed by kabirajs 
(20). While care-seeking is recognized as a complex behaviour influenced by many factors (21) in this rural population, kabirajs may offer a specialized niche in the rural healthcare market for provision of traditional treatments for perceived jaundeesh (8).

Most kabirajs inform patients that their symptoms will subside with their treatment after two to three weeks (22), a timespan which is co-incident with the self-limited natural history of most acute viral hepatitis. Patients report feeling that treatments, such as using amulets or washing away jaundeesh with spiritual water, have instant effects. While some treatments prescribed by kabirajs overlap with clinical recommendations, such as drinking plenty of fluids, most treatments prescribed for jaundice by kabirajs were non-biomedical. Some treatments were even potentially harmful, for example, one kabiraj recommended that his patient "wash his hands with water that has been standing for three days." Then, the kabiraj advised him to "to drink unpasteurized milk with herbal medicine." Kabirajs commonly advised patients to avoid meat and eggs, although protein intake is clinically recommended for optimal recovery (23). Practices such as these may hinder recovery or increase the risk of super-infection in patients already immunologically challenged by one infection.

Population-based studies that aim to use reported morbidities to detect hepatitis-like illnesses may benefit from an understanding that jaundeesh in the absence of visible signs of icterus, reported by two-thirds of our respondents, may be commonly perceived in this population. Given the degree of community trust and extant explanatory mechanisms for this illness, kabirajs are likely to remain first-line care providers for perceived jaundeesh in rural communities. Inclusion of kabirajs for health education and training may be useful to improve patient outcomes through referral of patients for whom immediate referral to clinics is warranted, such as cases of possible hepatitis $\mathrm{E}$ infections in pregnancy, where the prognosis is exceptionally poor and where clinical supportive management may reduce the risk of mortality (4).

This study was carried out on a random selection of rural residents and kabirajs in Matlab, an area of Bangladesh, generally representative of rural Bangladesh. Findings may be culturally unique to this study population, although anecdotal reports from Nepal suggest that similar local definitions are used for the word jaundice (Labrique, personal communication, 2012). Although additional interviews with respondents reporting jaundeesh as well as clinical signs of icterus might have allowed for comparison, this study focused on perceptions of jaundeesh among those lacking icterus in order to highlight local illness perceptions that may inform future population-based studies on reported illness symptoms.

\section{Conclusions}

Jaundeesh/holde palong is commonly recognized in this rural Bangladesh population as a distinct syndrome among anicteric respondents. Jaundeesh is associated with a number of signs and symptoms, some of which differ from the biomedical definition of jaundice. In this setting, kabirajs are the main care providers for jaundeesh and offer multiple treatment options, ranging from benign rituals to potentially harmful practices. Further research on the aetiology of hepatitis-like illness in this population should account for the local perceptions of the disease entity known as jaundeesh. An understanding of the cultural perceptions of jaundeesh may inform future population-based epidemiological surveys for surveillance of reported symptoms in populations of rural Bangladesh. These data may also inform national programmes for hepatitis prevention and treatment strategies, such as introduction of hepatitis B vaccination, and improve public-health strategies for surveillance, detection, and response.

\section{ACKNOWLEDGEMENTS}

This study was funded by NIH-NIAID grant (1 R01 AI51/31/2004). icddr,b acknowledges with gratitude the commitment of NIH-NIAD, USA to its research efforts. The authors would like to acknowledge the dedication and hard work of the HEV-Matlab field team (Bashiruddin Ahmad, S.R. Paul, A.B.M. Borhan Uddin, Nasrin Akter, Nurunnahar Lina, and Sabina Akhter) as well as the administration and staff of icddr,b for their support.

\section{REFERENCES}

1. Khan WI, Sultana R, Rahman M, Akhter H, Haq JA, Ali L et al. Viral hepatitis: recent experiences from serological studies in Bangladesh. Asian Pac J Allergy Immunol 2000;18:99-103.

2. Labrique AB, Thomas DL, Stoszek SK, Nelson KE. Hepatitis E: an emerging infectious disease. Epidemiol Rev 1999;21:162-79.

3. Labrique AB, Zaman K, Hossain Z, Saha P, Yunus M, Hossain A et al. Epidemiology and risk factors of incident hepatitis E virus infections in rural Bangladesh. Am J Epidemiol 2010;172:952-61.

4. Labrique AB, Sikder SS, Krain LJ, West KP, Jr., Chris- 
tian P, Rashid M et al. Hepatitis E, a vaccine-preventable cause of maternal deaths. Emerg Infect Dis 2012;18:1401-4.

5. Gurley ES, Halder AK, Streatfield PK, Sazzad HM, Huda TM, Hossain MJ et al. Estimating the burden of maternal and neonatal deaths associated with jaundice in Bangladesh: possible role of hepatitis $\mathrm{E}$ infection. Am J Public Health 2012;102:2248-54.

6. Labrique A. The epidemiology of hepatitis $\mathrm{E}$ virus infections in rural Bangladesh. Baltimore, MD: Johns Hopkins Bloomberg School of Public Health, 2007:2978 (Dissertation).

7. Purdy MA, Carson D, McCaustland KA, Bradley DW, Beach MJ, Krawczynski K. Viral specificity of hepatitis $\mathrm{E}$ virus antigens identified by fluorescent antibody assay using recombinant HEV proteins. J Med Virol 1994;44:212-4.

8. Bhuiya A, editor. Health for the rural masses: insights from Chakaria. Dhaka: ICDDR,B, 2009. 125 p. (ICDDR,B monograph no. 8).

9. Mahmood SS, Iqbal M, Hanifi SMA, Wahed T, Bhuiya A. Are 'Village Doctors' in Bangladesh a curse or a blessing? BMC Int Health Hum Rights 2010;10:18.

10. Sikder SS, Labrique AB, Ullah B, Ali H, Rashid M, Mehra $S$ et al. Accounts of severe acute obstetric complications in rural Bangladesh. BMC Pregnancy Childbirth 2011;11:76.

11. Sarder AM, Chen LC. Distribution and characteristics of non-government health practitioners in a rural area of Bangladesh. Soc Sci Med A 1981;15:543-50.

12. Christian P, Bentley ME, Pradhan R, West KP, Jr. An ethnographic study of night blindness "ratauni" among women in the Terai of Nepal. Soc Sci Med 1998;46:879-89.

13. Moran AC, Winch PJ, Sultana N, Kalim N, Afzal KM, Koblinsky M et al.; Bangladesh PROJAHNMO Maternal Morbidity Study Group. Patterns of maternal care seeking behaviours in rural Bangladesh. Trop Med Int
Health 2007;12:823-32.

14. Koenig MA, Jamil K, Streatfield PK, Saha T, Al-Sabir A, El Arifeen S et al. Maternal health and care-seeking behavior in Bangladesh: findings from a national survey. Int Fam Plan Perspect 2007;33:75-82.

15. Karim A. Shamanism in Bangladesh. Asian Folklore Studies 1998;47:277-309.

16. Berendes S, Heywood P, Oliver S, Garner P. Quality of private and public ambulatory health care in low and middle income countries: systematic review of comparative studies. PLoS Med 2011;8:e1000433.

17. Rahman A, Moran A, Pervin J, Rahman A, Rahman M, Yeasmin S et al. Effectiveness of an integrated approach to reduce perinatal mortality: recent experiences from Matlab, Bangladesh. BMC Public Health 2011;11:914.

18. Beckingham IJ, Ryder SD. ABC of diseases of liver, pancreas, and biliary system. Investigation of liver and biliary disease. BMJ 2001;322:33-6.

19. Mishra S, Tiwari SK, Kakkar A, Pandey AK. Chemoprofiling of Andrographis paniculata (kalmegh) for its andrographolide content in Madhya Pradesh, India. Int J Pharma Bio Sci 2010;1. 5 p. (http://www.ijpbs. net/issue-2/78.pdf, accessed on 15 August 2013).

20. Afsana K. Disciplining birth: power, knowledge, and childbirth practices in Bangladesh. Dhaka: University Press Limited, 2005. 242 p.

21. Andersen RM. Revisiting the behavioral model and access to medical care: does it matter? J Health Soc Behav 1995;36:1-10.

22. Ashraf H, Alam NH, Rothermundt C, Brooks A, Bardhan $\mathrm{P}$, Hossain L et al. Prevalence and risk factors of hepatitis $\mathrm{B}$ and $\mathrm{C}$ virus infections in an impoverished urban community in Dhaka, Bangladesh. BMC Infect Dis 2010;10:208.

23. Francavilla R, Miniello VL, Brunetti L, Lionetti ME, Armenio L. Hepatitis and cholestasis in infancy: clinical and nutritional aspects. Acta Paediatr Suppl 2003;91:101-4. 